\title{
Evaluation of heat stress and cumulative incidence of acute kidney injury in sugarcane workers in Guatemala
}

\author{
Jaime Butler-Dawson ${ }^{1,2,3}$ - Lyndsay Krisher ${ }^{1,2} \cdot$ Hillary Yoder $^{4} \cdot$ Miranda Dally $^{1,2,3} \cdot$ Cecilia Sorensen $^{2,5}$. \\ Richard J. Johnson ${ }^{2,6}$. Claudia Asensio ${ }^{7}$. Alex Cruz • Evan C. Johnson ${ }^{4}$ - Elizabeth J. Carlton ${ }^{2,3} \cdot$ Liliana Tenney $^{1,2,3}$. \\ Edwin J. Asturias ${ }^{8,9,10} \cdot$ Lee S. Newman $1,2,3,11$
}

Received: 1 October 2018 / Accepted: 19 March 2019 / Published online: 17 April 2019

(c) The Author(s) 2019

\begin{abstract}
Objective Agricultural workers worldwide exposed to heat stress could be at the risk of kidney injury, which could lead to chronic kidney disease of an unknown origin $(\mathrm{CKDu})$. Hydration has been promoted as a key measure to reduce kidney injury. In the presence of a hydration intervention, the incidence of acute kidney injury (AKI) was calculated in a sugarcane worker population in Guatemala and several risk factors were evaluated.

Methods We measured kidney function at the beginning and end of the work shift at three time points in 517 sugarcane workers. We defined AKI as an increase in serum creatinine of $26.5 \mu \mathrm{mol} / \mathrm{L}$ or $50 \%$ or more from the pre-shift value. Associations between AKI and risk factors were examined, including interactions with hydration status.

Results The prevalence of dehydration post-shift (>1.020 specific gravity) was $11 \%$ in February, $9 \%$ in March, and $6 \%$ in April. Cumulative incidence of AKI was 53\% in February, 54\% in March, and 51\% in April. AKI was associated with increasing post-shift specific gravity, a dehydration marker, (OR 1.24, 95\% CI 1.02-1.52) and with lower electrolyte solution intake (OR 0.94, 95\% CI 0.89-0.99).

Conclusions Dehydration and insufficient electrolyte consumption are risk factors for AKI. However even well-hydrated sugarcane workers routinely experience AKI. While hydration is important and protective, there is a need to understand other contributors to risk of AKI and identify prevention strategies with these workers.
\end{abstract}

Keywords Agricultural workers $\cdot$ Kidney injury $\cdot$ Hydration

\section{Introduction}

Daily heat stress and dehydration may be associated with recurrent acute kidney injury (AKI) among agricultural workers around the world (Roncal-Jimenez et al. 2015). Workers who experience recurrent episodes of AKI may be at substantial risk for developing chronic kidney disease of unknown origin (CKDu), a newly described global epidemic (Johnson et al. in press; Vos et al. 2017), specifically in agricultural communities in low altitudes along the pacific coast of Central America (Hahn et al. 2017; Roncal-Jimenez

Electronic supplementary material The online version of this article (https://doi.org/10.1007/s00420-019-01426-3) contains supplementary material, which is available to authorized users.

Jaime Butler-Dawson

jaime.butler-dawson@ucdenver.edu

Extended author information available on the last page of the article et al. 2015). Any workers who regularly labor in hot conditions could be at the risk of kidney injury and CKDu, with profound implications for worker health, safety, and sustainable food production as global temperatures continue to rise (Glaser et al. 2016; Watts et al. 2017).

There are a number of potential causes of AKI among agricultural workers that could operate independently or in combination. These include (1) volume depletion and dehydration due to heat stress and high physical demand, (2) subclinical or clinical rhabdomyolysis due to muscle damage from extreme labor, (3) tobacco use, and (4) non-steroidal anti-inflammatory drug (NSAID) use (Butler-Dawson et al. 2018; Correa-Rotter et al. 2014; Hodgson et al. 2017; Speeckaert et al. 2013). Agricultural work, specifically sugarcane cutting, involves intense labor in very hot temperatures (Butler-Dawson et al. 2018; Correa-Rotter et al. 2014; Crowe et al. 2013). Recent studies have shown heat stress to be a risk factor for cross-shift kidney damage in agricultural 
workers in Central America and the U.S. (Garcia-Trabanino et al. 2015; Mix et al. 2017; Moyce et al. 2017; Sorensen et al. 2019). Dehydration has also been identified as a risk factor for kidney damage among sugarcane workers laboring under hot conditions (Roncal-Jimenez et al. 2015). However, most studies have not found direct associations between dehydration and kidney injury (Butler-Dawson et al. 2018; Laws et al. 2016; Moyce et al. 2017).

There are few studies geared towards interventions to address the risk of repeat heat stress and dehydration in sugarcane worker populations. One study conducted in El Salvador implemented a water, rest, shade (WRS) intervention halfway through the 6-month sugarcane harvest (Bodin et al. 2016; Wegman et al. 2018). These authors reported that the WRS intervention resulted in a reduction in dehydration symptoms and a smaller decline in kidney function across the work shift compared to sugarcane workers not receiving the intervention. While the results suggest a protective effect of increased water consumption, direct associations between hydration and kidney function were not examined. Therefore, it remains unclear whether increasing water intake can reduce AKI events across the work shift among agricultural workers.

We implemented an enhanced hydration intervention among a large number of sugarcane workers in southwest Guatemala to evaluate the effect of improved hydration on cumulative incidence of AKI and to determine the role of other risk factors. We hypothesized that kidney injury, occurring at a sub-clinical level due to heat stress while performing intense labor in hot conditions, would be associated with risk factors like dehydration, physical workload, use of nephrotoxic medications such as NSAIDs, tobacco use, and sugary beverage intake (Correa-Rotter et al. 2014; Garcia-Arroyo et al. 2016; Speeckaert et al. 2013; Weiner et al. 2013).

\section{Methods}

\section{a. Study population}

For this prospective longitudinal cohort study, we collected demographic, clinical, and laboratory data to evaluate kidney function and risk factors in 517 sugarcane workers from ten field work groups. Work groups consisted of sugarcane cutters and production workers employed for the 2016-2017 harvest season at a sugarcane mill in Guatemala owned by Pantaleon, an agribusiness and producer of sugar in Central America (Fig. 1). Cane cutters cut and pile sugarcane while production workers perform tasks such as cutting and planting cane seed. Work setting, worker population, and work practice details have been previously described (Butler-Dawson et al. 2018) and more detail is provided in the supplemental material.

All study participants ( $\geq 18$ years) were male, screened for employment between August and November, and started the season with an estimated glomerular

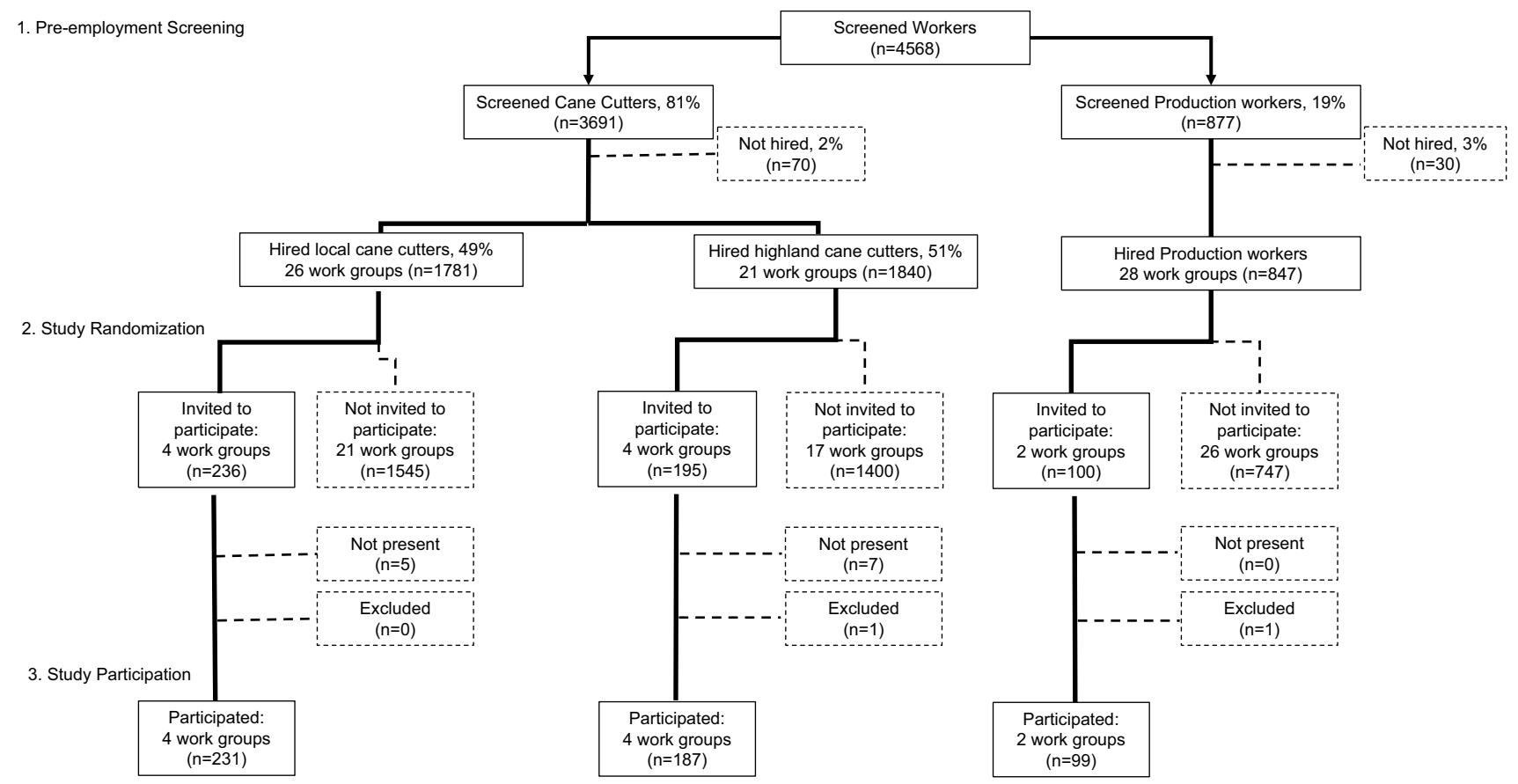

Fig. 1 Flowchart of pre-employment screening and study recruitment 
filtration rate $(\mathrm{eGFR}) \geq 60 \mathrm{ml} / \mathrm{min} / 1.73 \mathrm{~m}^{2}$ and no major illnesses that might affect their ability to work.

b. Study design

Data were collected before and after three work shifts for each work group during three consecutive months, February, March, and April 2017. In January 2017, prior to the start of data collection, workers from ten randomly selected work groups were recruited and consented for this study. We used stratified random sampling to construct the list of ten work groups stratified by job type, eight cane cutter work groups and two production work groups (Fig. 1). Among the cane cutter work groups, we also stratified by home residence. Four local resident work groups and four highland resident work groups were selected. Workers in the local resident work groups live in local communities in the coastal lowland area surrounding the sugarcane fields. Workers in the highland resident work groups are from the highland regions at higher altitudes, however, they live in dormitories at the mill for the duration of the harvest. Workers in the production work groups are only from the local communities.

Data were available on baseline demographics for the worker population from which study participants were drawn. Pantaleon routinely collects pre-employment survey, clinical and laboratory data, including creatinine measures on the field workers, and we were able to leverage those data to assess differences between workers selected for the study and workers not selected. There were no significant differences in age $(p=0.49)$, home residence $(p=0.15)$, baseline BMI $(p=0.32)$, and baseline eGFR $(p=0.29)$ between selected workers and workers not selected.

c. Study intervention

One of the goals of our study was to facilitate actionable workplace policies and practices by determining how well we could prevent AKI by maximizing hydration. To accomplish this, we enhanced the current WRS program used by the company since 2009 (supplemental material). The enhanced WRS intervention was provided to all study participants and included two components: (1) amplifying the existing worker education program on the importance of WRS, and (2) providing a "wellness incentive" based on workers' hydration status at the start and end of the work shift. At the time of consent and throughout the study, study personnel and trained Pantaleon nurse aides provided additional education through face-to-face communication, posters, and pocket urine color charts (Human Hydration, LLC, Hampton, VA) for self-evaluation of hydration status, translated to Spanish and adapted for low literacy (see supplemental material). For the wellness incentive, all study participants were offered incentives on their three study days. The worker received tokens if he started the study work shift hydrated (pre-shift urinary specific gravity was $\leq 1.020$ ) (Perrier et al. 2017) or if he maintained or improved his hydration status across the work shift $(<1 \%$ body weight loss) (Webb et al. 2016). If a worker was not considered hydrated, he was encouraged to drink additional water and take more rest breaks. The workers entered tokens into a raffle for chances to win small non-monetary prizes (i.e., soap, towels, socks, soccer balls, plastic reusable food containers, and water bottles) at the end of each study day.

d. Study ethics review

Ethics review and approval for the study was done by the Colorado Multiple Institution Review Board (COMIRB) and in Guatemala by the Comite de Etica, Facultad de Medicina, Universidad Francisco Marroquin-Hospital Universitario Esperanza. All participants provided written informed consent at the time of enrollment.

e. Data collection

\section{Pre-employment screening}

To examine baseline health of the workers, we utilized preemployment data, including creatinine measures that were collected by Pantaleon between August and November 2016. Serum creatinine was collected from a blood draw and sent to an independent, licensed clinical laboratory (Herrera Llerandi laboratory, Guatemala City, Guatemala). Creatinine values were performed in duplicate using the Creatinine Jaffe Generation 2 method. Survey data included number of previous harvests, self-reported diabetes, home residence, and job type. Clinical data included age, blood pressure (at least 3 min of seated rest before the measurement), weight, and height. Hypertension was defined as systolic blood pressure $\geq 140 \mathrm{mmHg}$ and/or diastolic blood pressure $\geq 90 \mathrm{mmHg}$. We obtained written consent from study participants to link their pre-employment data to the study data.

\section{Pre- and post-shift sampling}

Body weight, urinary specific gravity, and point-of-care (POC) creatinine were collected for each participant prior to the start of the work shift (5-9 am) at three time points. Body weight was measured using a scale (Microlife WS 100 digital scale, Clearwater, FL) that was placed on a stable platform and calibrated prior to each data collection session. Workers were weighed in work clothing with shin guards removed. To adjust for the additional weight of the clothes at the end of the day due to sweat and dirt, we calculated a correction factor (supplemental material) and subtracted the correction factor from the worker's post-shift weight. 
Specific gravity was measured using a digital refractometer (ATAGO PAL-10S digital refractometer, Tokyo, Japan) within 10 min of workers providing a urine sample before and after the work shift, according to manufacturer's instructions. To measure creatinine pre- and post-shift, blood was collected by finger prick and read instantly in the field using the Nova ${ }^{\circledR}$ Statscan (Stat Sensor Creatinine Meter, Nova Biomedical Corporation, Waltham, MA, USA).

The aforementioned pre-shift measures were repeated at the end of the work shift (1-4 pm for production workers and 4-7 pm for cane cutters). In addition, workers completed an interviewer-administered post-shift survey (supplemental material). The survey was piloted in a sub-sample of the target population and was delivered by Spanish-speaking interviewers. The survey included questions about workers' past 24-h behaviors including heat- and pain-related symptoms during work shift (yes or no), number of cigarettes since they woke up, number of sugary beverages since they woke up, and number of alcoholic beverages consumed the previous night. The survey also included a detailed 24-h recall of oral and parenteral medications, vitamins, and supplements (yes or no and count for each type) and reasons why they took these items. We showed participants pictures of locally available pills and injectables as visual prompts. We created a variable, "NSAID use" (yes or no), based on the participant's responses including use of ibuprofen, aspirin, diclofenac, naproxen, or local brands that were identified as an NSAID.

\section{Assessment of heat exposure}

On each study day, we collected wet bulb globe temperature (WBGT) in the sugarcane field where the study group was working during work shift hours (3M QUESTemp 34, Thermal Environmental Monitor, St. Paul, MN, USA). WBGT is a measure of heat exposure and is used by the National Institute for Occupational Safety and Health (NIOSH) and the International Organization for Standardization (ISO) to establish guidelines for work/rest cycles in different environments (OSHA 2017). The WBGT meter provided output on the average and maximum WBGT for each study day. Of note, on the last study day in April, the WBGT meter only recorded for $40 \mathrm{~min}$, from 2:00 to 2:40 pm. This 40-min WBGT measure could overestimate the average WBGT and potentially underestimate the maximum WBGT, so we excluded that day's measurements.

\section{Assessment of acute kidney injury}

The development of AKI was the primary health outcome variable. We identified workers with AKI based on their pre- and post-shift POC creatinine measures using the Kidney Disease: Improving Global Outcomes (KDIGO) criteria (KDIGO 2012). This criterion defines AKI as either an increase in the creatinine level by $\geq 26.5 \mu \mathrm{mol} / \mathrm{L}$ or an increase in the creatinine level to $\geq 1.5$ times the pre-shift level. Based on previous comparisons between post-shift POC creatinine measures and venipuncture creatinine measures, we applied an adjustment factor of 0.7775 to all the POC creatinine values (Griffin et al. 2018). Adjusted POC creatinine was used to identify workers with AKI and calculate eGFR using the Chronic Kidney Disease Epidemiology Collaboration (CKD-EPI) equation for all participants (Levey and Stevens 2010).

\section{Assessment of hydration status and electrolyte solution intake}

Hydration status was assessed in two ways: (1) percent change in body weight from pre- to post-shift, and (2) preand post-shift urine specific gravity. In addition, in the postshift survey, we asked participants to quantify the number of liters of water and number of $500 \mathrm{ml}$ electrolyte solution packets they had consumed since waking up that day.

\section{Assessment of physical work intensity}

We measured workers' levels of physical exertion using three variables: work shift hours, productivity, and productivity $z$-scores. The length of their work shift was based on the time the worker finished the pre-shift data collection and started the post-shift data collection. Productivity data were provided by Pantaleon on each cane cutter's daily amount of cane they cut. These data included the number of tons of cane cut on each study day and the day prior to each study day for each cane cutter. Individual productivity $z$-scores were reported relative to an individual's daily average tons cut during the entire season. The $z$-score was calculated using the following formula: (individual workers study day productivity - individual season average productivity)/individual standard deviation. Positive $z$-scores indicate aboveaverage productivity, and negative scores indicate belowaverage productivity. In addition, participants reported the number of rest breaks (longer than $15 \mathrm{~min}$ ), including lunch, that they took during the day's shift.

\section{f. Statistical analysis}

Participant characteristics (i.e., demographics, behavior, and clinical data) are described for both the cane cutters and production workers. The majority of these data were not normally distributed and are expressed as the median and interquartile range (IQR). Pre- and post-shift specific gravity were placed into three clinical categories (maximally dilute, <1.005; hydrated, 1.005-1.020; and dehydrated, $>1.020$ ) to present the hydration status across the 3 months. 
To address our hypothesis that AKI can be explained by certain risk factors, we estimated the associations between pre-specified risk factors and AKI using mixedeffects logistic regression analysis with all the workers combined. We began our statistical analyses by examining $\mathrm{AKI}$ in relation to each potential risk factor using linear mixed models with random intercept for each worker to account for repeated measures. We then examined correlations between WBGT and several variables that might be related to WBGT including work shift hours, post-shift specific gravity, electrolyte intake, and productivity using Spearman's rank correlation coefficient $(r)$. For the multivariable analysis, we included all variables that had $p$ value $<0.10$ in the univariate models and age, hypertension (yes or no), and baseline eGFR as covariates that were selected as a priori known risk factors for reduced kidney function. In the final multivariable model, both pre- and post-shift urinary specific gravity were included in the model, since they were only moderately correlated and not collinear $(r=0.42$, $p<0.01$ ). However, maximum WBGT and average WBGT were strongly correlated $(r=0.62, p<0.001)$, so we ran the model with only average WBGT. Job type was significant in the univariate analysis; however, since job type was highly associated with specific gravity, one of our main exposures of interest, we did not include it in the final model.

We were additionally interested in the extent to how dehydration modifies the relationship between certain behavioral risk factors and AKI. Based on previous literature, we examined the following risk factors: smoking (yes or no), sugary beverage intake (per 1 drink increase) and NSAID use (yes or no) (Butler-Dawson et al. 2018; Garcia-Arroyo et al. 2016; Lunyera et al. 2016). We fit a logistic regression model with an interaction term between each individual explanatory behavioral variable and post-shift urinary specific gravity on the odds of developing an AKI. We then included any significant interaction terms in the final multivariable model. A two-sided $p$ value of $<0.05$ was considered as the threshold for statistical significance. All the analyses were performed using SAS Release 9.4 (SAS Institute, Cary, NC).

\section{Results}

\section{Study participation}

Of 534 workers who were in the selected ten work groups, 522 were present the day of consent and enrolled in the study. Three workers who consented were not present on any of the study days and two workers were excluded from the analysis because they were supervisors and did not perform similar tasks. Therefore, we included 517 workers in this analysis. In total, 425 workers (82\%) participated at all three time points, 66 workers (13\%) participated at two time points, and 27 (5\%) participated at one time point. In terms of study attrition, 32 workers (6\%) dropped out of the workforce during the study, $54(10 \%)$ were absent during their study day, and 10 workers (2\%) refused to participate at one time point (8 in March and 2 in April). Participants who left the workforce early ( 28 cane cutters and 4 production workers) were significantly more likely to be younger (26 vs. 28 years, $p=0.04$ ) and to be a highland worker compared to a local worker (10\% and 4\%, $p<0.01$, respectively). Participants who were absent were more likely to be cane cutters than production workers $(12 \%$ vs. $3 \%, p<0.01)$. Participants who refused to participate were more likely to be highland workers than local workers ( $4 \%$ vs. $1 \%, p<0.01)$. Study participants who left early, were absent, or refused were not significantly different in hypertension status or kidney function compared to those who remained in the study (supplemental material).

\section{Heat exposure}

During the eight February study days, the average WBGT ranged from 29.5 to $32.9^{\circ} \mathrm{C}$ and the maximum WBGT ranged from 31.7 to $36.4{ }^{\circ} \mathrm{C}$. During eight March days, the average WBGT ranged from 28.2 to $32.2{ }^{\circ} \mathrm{C}$ and the maximum WBGT ranged from 31.2 to $35.5^{\circ} \mathrm{C}$. During the seven April days, the average WBGT ranged from 30.9 to $32.0^{\circ} \mathrm{C}$ and maximum WBGT ranged from 33.5 to $35.5^{\circ} \mathrm{C}$. To put into context, the Occupational Safety and Health Administration (OSHA) heat exposure threshold for work/ rest regimens for "very heavy" work at $30.0{ }^{\circ} \mathrm{C}$ is for workers to work 25\% and rest 75\% per hour (Crowe et al. 2013; OSHA 2017). Only 4 out of the 23 study days had an average WBGT below $30.0{ }^{\circ} \mathrm{C}$.

a. Cane cutter results.

\section{Baseline demographics and clinical data}

In total, 418 cane cutters participated in the study. Cane cutters had a median age of 28 years (IQR 24-35) and a median BMI of 23 (IQR 21-25), Table 1. Fifty-five percent of the cutters were from the local area. Cane cutters reported having worked a median of seven harvest years (IQR 4-12). At baseline, $2 \%$ of the cutters had hypertension and none of them reported having diabetes. 
Table 1 Cane cutter characteristics at three study time points in Guatemala, 2017

\begin{tabular}{|c|c|c|c|}
\hline \multicolumn{3}{|l|}{ Pre-employment Screening } & \multirow{2}{*}{$\begin{array}{l}\text { Characteristics, } \\
\text { median (IQR), } n(\%)\end{array}$} \\
\hline Total number of workers & & & \\
\hline Baseline age, years & & & $28(24-35)$ \\
\hline Baseline body mass index, $\mathrm{kg} / \mathrm{m}^{2}$ & & & $22.6(21.2-24.5)$ \\
\hline Hypertension & & & $8(2 \%)$ \\
\hline \multicolumn{4}{|l|}{ Home residence } \\
\hline Local & & & $231(55 \%)$ \\
\hline Highland & & & $187(45 \%)$ \\
\hline Study time points & February & March & April \\
\hline Participating workers $(N)$ & 411 & 355 & 373 \\
\hline \multicolumn{4}{|l|}{ Hydration and electrolytes } \\
\hline Pre-shift body weight (kg) & $59(54,64)$ & $58(54,63)$ & $58(54,63)$ \\
\hline Body weight, percent change & $0.3 \%(-1.4,1.8)$ & $0.3 \%(-1.6,1.8)$ & $1.1 \%(-0.6,2.8)$ \\
\hline Pre-shift urinary specific gravity & $1.003(1.002,1.006)$ & $1.004(1.002,1.007)$ & $1.004(1.002,1.008)$ \\
\hline Maximally dilute $(<1.005)$ & $279(68 \%)$ & $207(58 \%)$ & $188(51 \%)$ \\
\hline Normal (1.005-1.020) & $116(28 \%)$ & $135(38 \%)$ & $177(48 \%)$ \\
\hline Dehydrated (>1.020) & $15(4 \%)$ & $13(4 \%)$ & $7(2 \%)$ \\
\hline Post-shift urinary specific gravity & $1.002(1.001,1.004)$ & $1.002(1.001,1.004)$ & $1.002(1.001,1.004)$ \\
\hline Maximally dilute $(<1.005)$ & $335(82 \%)$ & $281(79 \%)$ & $290(78 \%)$ \\
\hline Normal (1.005-1.020) & $68(17 \%)$ & $68(19 \%)$ & $74(20 \%)$ \\
\hline Dehydrated (> 1.020) & $7(2 \%)$ & $7(2 \%)$ & $7(2 \%)$ \\
\hline Urinary-specific gravity, percent change ${ }^{a}$ & $-0.07 \%(-0.32,0.07)$ & $-0.14 \%(-0.43,0.01)$ & $-0.10 \%(-0.50,0.00)$ \\
\hline Electrolytes, number of $500 \mathrm{ml}$ packets & $6(5,8)$ & $6(5,7)$ & $6(5,8)$ \\
\hline Water intake, liters & $15(15,17)$ & $15(15,17)$ & $15(15,16)$ \\
\hline \multicolumn{4}{|l|}{ Physical work intensity } \\
\hline Rest breaks & $4(3,4)$ & $4(3,4)$ & $4(3,4)$ \\
\hline Work shift, hours & $10.42(9.90,10.93)$ & $10.90(10.37,11.53)$ & $10.45(9.73,11.10)$ \\
\hline Productivity, prior day, tons/day & $5.7(4.6,7.0)$ & $5.3(3.6,7.4)$ & $5.8(4.8,6.9)$ \\
\hline Productivity, study day, tons/day & $5.4(4.3,6.9)$ & $6.0(4.7,7.9)$ & $5.1(4.1,6.4)$ \\
\hline Productivity $z$-score ${ }^{\mathrm{b}}$, study day & $-0.20(-0.94,0.44)$ & $-0.58(-1.12,-0.05)$ & $-0.39(-0.81,0.28)$ \\
\hline \multicolumn{4}{|l|}{ Heat exposure } \\
\hline Average WBGT, ${ }^{\circ} \mathrm{C}$ & $32.5(31.0,32.5)$ & $30.9(30.7,32.2)$ & $31.3(31.1,31.4)$ \\
\hline Maximum WBGT, ${ }^{\circ} \mathrm{C}$ & $36.4(33.8,36.4)$ & $35.1(34.4,35.1)$ & $34.1(33.7,34.4)$ \\
\hline \multicolumn{4}{|l|}{ Study day behaviors } \\
\hline Smoked cigarette (count range) & $12(3 \%),(1-6)$ & $13(4 \%),(1-3)$ & $19(6 \%),(1-5)$ \\
\hline NSAID use (count range) & $32(8 \%),(1-9)$ & $9(2 \%),(1-5)$ & $15(4 \%),(1-3)$ \\
\hline Sugary drink consumption (count range) & $99(24 \%),(1-10)$ & $89(26 \%),(0.5-5)$ & $138(39 \%),(1-6)$ \\
\hline Alcohol consumption (count range) & $4(1 \%),(2-5)$ & $12(3 \%),(1-4)$ & $7(2 \%),(1-7)$ \\
\hline \multicolumn{4}{|l|}{ Study day symptoms ${ }^{\mathrm{c}}$} \\
\hline Headache & $13(3 \%)$ & $3(1 \%)$ & $11(3 \%)$ \\
\hline Fever & $2(<1 \%)$ & 0 & $2(<1 \%)$ \\
\hline Urination pain & 0 & $1(<1 \%)$ & $1(<1 \%)$ \\
\hline Vomit & 0 & $1(<1 \%)$ & 0 \\
\hline Weakness & 0 & $2(<1 \%)$ & $1(<1 \%)$ \\
\hline Dry mouth & $2(<1 \%)$ & $2(<1 \%)$ & 0 \\
\hline Swelling of hands and legs & $1(<1 \%)$ & $6(2 \%)$ & 0 \\
\hline Upper back pain & 0 & $2(<1 \%)$ & $2(<1 \%)$ \\
\hline Lower back pain & $5(1 \%)$ & $3(1 \%)$ & $3(1 \%)$ \\
\hline Breathing difficulty & $4(1 \%)$ & $2(<1 \%)$ & $2(<1 \%)$ \\
\hline
\end{tabular}

NSAID non-steroidal anti-inflammatory, WBGT wet bulb globe temperature

Hypertension, defined as systolic blood pressure $\geq 140 \mathrm{mmHg}$ and/or diastolic blood pressure $\geq 90 \mathrm{mmHg}$

${ }^{a}$ Negative value shows a decrease in specific gravity across the work shift

${ }^{\mathrm{b}} \mathrm{Z}$-score calculation: individual workers study day productivity—individual average productivity/individual standard deviation 
Table 1 (continued)

${ }^{c}$ No participants reported the following symptoms: cramping in legs or arms, diarrhea, heart palpitations, dizzy, and ear pain

\section{Hydration status and electrolyte solution intake}

As shown in Table 1, body weight was maintained across the work shift and percent change in body weight across the shift was slightly positive at all three points. Results from the pre-shift urinary specific gravity show that almost all of the workers started their work shift well hydrated (96\% in February and March, and $98 \%$ in April). Notably, at the end of the shift, almost all of the workers had maintained hydration status. Workers reported drinking a median of three liters (six $500 \mathrm{ml}$ packets) of electrolyte solution and drinking a median of 15 liters of water.

\section{Cumulative incidence of AKI and kidney function}

Among the 418 cane cutters, 324 (78\%) had at least one episode of AKI over the three work shifts. Thirty-eight percent of workers had one AKI, 28\% of workers had two AKIs, and $11 \%$ had three AKIs. The cumulative incidence of AKI over a work shift was $47 \%$ in February, $51 \%$ in March and $45 \%$ in April (Table 2). The percent of workers with a stage 2 AKI doubled across the season, from 7 to $14 \%$, defined as post-shift creatinine 2.0-2.9 times higher than pre-shift (KDIGO 2012).

The median eGFR at baseline was $116 \mathrm{ml} / \mathrm{min} / 1.73 \mathrm{~m}^{2}$ and the median pre-shift eGFR was approximately 130 at all three time points. Almost all of the workers (92-94\%) had a normal eGFR at the start of the work shift $(\geq 90)$. One worker had an eGFR $<60$ at all three time points and two workers had $<60$ at two time points.

\section{Physical work intensity}

Workers were in the field approximately $10 \mathrm{~h}$ at each time point, including rest periods. They reported taking a median of four rest breaks of at least 15 min duration. The median tons of cane cut by each worker on the study days were 5.7 tons in February, 5.3 tons in March, and 5.8 tons in April. The median tons cut on the day prior to the study day was 5.4 tons in February, 6.0 tons in March, and 5.1 tons in April.

\section{Behavioral risk factors and symptoms}

Very few workers $(<9 \%)$ reported smoking any cigarettes the day of the study, taking a NSAID in the last $24 \mathrm{~h}$, or consuming alcoholic beverages the night before. One-quarter of the workers reported drinking a sugary beverage the day of the study in February and March, with an increase to $40 \%$ in April. Very few of the workers reported symptoms on the study day, with headache being the most frequently reported symptom, at $3 \%$ in both February and April.

Table 2 Kidney function of the cane cutters at the three study time points in Guatemala, 2017

\begin{tabular}{|c|c|c|c|}
\hline $\begin{array}{l}\text { Kidney function } \\
\text { Pre-shift }\end{array}$ & $\begin{array}{l}\text { February } \\
n=412\end{array}$ & $\begin{array}{l}\text { March } \\
n=355\end{array}$ & $\begin{array}{l}\text { April } \\
n=373\end{array}$ \\
\hline Creatinine, $\mu \mathrm{mol} / \mathrm{L}$, median (IQR) & $59.23(50.39,71.60)$ & $57.46(47.74,69.84)$ & $57.46(47.74,68.95)$ \\
\hline eGFR, $\mathrm{ml} / \mathrm{min} / 1.73 \mathrm{~m}^{2}$, median (IQR) & $129.04(118.20,140.40)$ & $130.87(116.76,142.42)$ & $131.79(118.99,142.51)$ \\
\hline \multicolumn{4}{|l|}{ Kidney disease stages, $n(\%)$} \\
\hline$>90$ eGFR & $380(92 \%)$ & $331(93 \%)$ & $348(94 \%)$ \\
\hline 60-90 eGFR & $25(6 \%)$ & $20(6 \%)$ & $19(5 \%)$ \\
\hline 30-60 eGFR & $5(1 \%)$ & $4(1 \%)$ & $5(1 \%)$ \\
\hline 15-30 eGFR & $1(<1 \%)$ & 0 & 0 \\
\hline Cross-shift & $n=411$ & $n=348$ & $n=370$ \\
\hline Change in creatinine, median (IQR) & $0.27(0.18,0.38)$ & $0.28(0.18,0.40)$ & $0.27(0.18,0.38)$ \\
\hline Acute kidney injury $^{\mathrm{a}}, n(\%)$ & $191(47 \%)$ & $178(51 \%)$ & $166(45 \%)$ \\
\hline \multicolumn{4}{|l|}{ AKI categories ${ }^{\mathrm{b}}, n(\%)$} \\
\hline Stage 1 & $176(92 \%)$ & $155(87 \%)$ & $140(85 \%)$ \\
\hline Stage 2 & $13(7 \%)$ & $20(11 \%)$ & $24(14 \%)$ \\
\hline Stage 3 & $2(1 \%)$ & $3(2 \%)$ & $2(1 \%)$ \\
\hline
\end{tabular}

$e G F R$ estimated glomerular filtration rate, $I Q R$ interquartile range

${ }^{\mathrm{a}}$ Increase of post-shift creatinine by $\geq 26.5 \mu \mathrm{mol} / \mathrm{L}$ or 1.5 times pre-shift creatinine

${ }^{\mathrm{b}}$ Stage 1: $1.5-1.9$ times pre-shift or $\geq 26.5 \mu \mathrm{mol} / \mathrm{L}$ increase. Stage 2: 2-2.9 times pre-shift. Stage 3: 3 times pre-shift or $354 \mu \mathrm{mol} / \mathrm{L}$ increase 
b. Production worker results

Baseline demographics and clinical data

In total, 99 production workers participated. Production workers had a median age of 30 years (IQR: 24-38) and a

Table 3 Production worker characteristics at the three study time points in Guatemala, 2017

\begin{tabular}{|c|c|c|c|}
\hline Pre-employment screening & \multicolumn{3}{|c|}{ Characteristics, median (IQR), $n(\%)$} \\
\hline Total number of workers & \multicolumn{3}{|l|}{99} \\
\hline Baseline age, years & \multicolumn{3}{|l|}{$30(24-38)$} \\
\hline Baseline body mass index, $\mathrm{kg} / \mathrm{m}^{2}$ & \multicolumn{3}{|l|}{$24.0(21.6-25.6)$} \\
\hline Hypertension & \multicolumn{3}{|l|}{$8(9 \%)$} \\
\hline Study time points & February & March & April \\
\hline Participating workers $(N)$ & 98 & 95 & 95 \\
\hline \multicolumn{4}{|l|}{ Hydration and electrolytes } \\
\hline Pre-shift body weight (kg) & $57(54,62)$ & $59(54,64)$ & $59(54,66)$ \\
\hline Body weight, percent change & $-0.6 \%(-1.6,0.8)$ & $-0.6 \%(-1.4,0.3)$ & $0.2 \%(-0.9,1.7)$ \\
\hline Pre-shift urinary specific gravity & $1.016(1.01,1.02)$ & $1.014(1.007,1.019)$ & $1.008(1.004,1.013)$ \\
\hline Maximally dilute $(<1.005)$ & $12(13 \%)$ & $13(14 \%)$ & $28(29 \%)$ \\
\hline Normal (1.005-1.020) & $61(64 \%)$ & $59(62 \%)$ & $67(71 \%)$ \\
\hline Dehydrated (>1.020) & $23(24 \%)$ & $23(24 \%)$ & $0(0 \%)$ \\
\hline Post-shift urinary specific gravity & $1.021(1.014,1.025)$ & $1.014(1.005,1.024)$ & $1.009(1.002,1.019)$ \\
\hline Maximally dilute $(<1.005)$ & $15(15 \%)$ & $21(23 \%)$ & $39(41 \%)$ \\
\hline Normal (1.005-1.020) & $32(33 \%)$ & $38(39 \%)$ & $34(36 \%)$ \\
\hline Dehydrated $(>1.020)$ & $51(52 \%)$ & $36(38 \%)$ & $22(23 \%)$ \\
\hline Urinary-specific gravity, percent change $^{\mathrm{a}}$ & $0.34 \%(-0.14,0.91)$ & $0.16 \%(-0.40,0.69)$ & $0.10 \%(-0.40,1.19)$ \\
\hline Electrolytes, number of $500 \mathrm{ml}$ packets & $1(0,1)$ & $1(0,1)$ & $2(2,3)$ \\
\hline Water intake, liters & $5(4,6)$ & $5(4,7)$ & $5(4,6)$ \\
\hline \multicolumn{4}{|l|}{ Physical work intensity } \\
\hline Rest breaks & $2(1,3)$ & $2(2,3)$ & $2(2,3)$ \\
\hline Work shift, hours & $8.00(7.22,8.65)$ & $7.78(7.38,9.05)$ & $7.92(6.90,8.18)$ \\
\hline \multicolumn{4}{|l|}{ Heat exposure } \\
\hline Average WBGT, ${ }^{\circ} \mathrm{C}$ & $32.4(29.5,32.4)$ & $29.9(28.2,29.9)$ & $33.3(32.0,33.3)$ \\
\hline Maximum WBGT, ${ }^{\circ} \mathrm{C}$ & $34.7(31.7,34.7)$ & $32.6(31.2,32.6)$ & $33.8(33.8,35.2)$ \\
\hline \multicolumn{4}{|l|}{ Study day behaviors } \\
\hline Smoked cigarette (count range) & $4(4 \%)(1-10)$ & $3(4 \%)(1)$ & $3(4 \%)(1-3)$ \\
\hline NSAID use (count range) & $4(4 \%)(1-3)$ & $1(1 \%)(1)$ & $4(4 \%)(1-2)$ \\
\hline Sugary drink consumption (count range) & $55(56 \%)(1-3)$ & $33(38 \%)(1-3)$ & $40(48 \%)(1-3)$ \\
\hline Alcohol consumption (count range) & $3(4 \%)(2-5)$ & $3(4 \%)(2-3)$ & $1(1 \%)(2)$ \\
\hline \multicolumn{4}{|l|}{ Study day symptoms ${ }^{\mathrm{b}}$} \\
\hline Headache & $1(1 \%)$ & $1(1 \%)$ & $1(1 \%)$ \\
\hline Cramping in legs or arms & $1(1 \%)$ & 0 & 0 \\
\hline Urination pain & $1(1 \%)$ & 0 & $1(1 \%)$ \\
\hline Faint & $1(1 \%)$ & 0 & 0 \\
\hline Dry mouth & $9(9 \%)$ & $1(1 \%)$ & $1(1 \%)$ \\
\hline Upper back pain & $2(2 \%)$ & 0 & 0 \\
\hline Lower back pain & $3(3 \%)$ & $1(1 \%)$ & $1(1 \%)$ \\
\hline
\end{tabular}

NSAID non-steroidal anti-inflammatory, WBGT wet bulb globe temperature. Hypertension, defined as systolic blood pressure $\geq 140 \mathrm{mmHg}$ and/ or diastolic blood pressure $\geq 90 \mathrm{mmHg}$

swelling of hands and legs

${ }^{a}$ Negative value shows a decrease in specific gravity across the work shift

${ }^{\mathrm{b}}$ No participants reported the following symptoms: fever, diarrhea, heart palpitations, dizzy, vomit, ear pain, breathing difficulty, and 
median BMI of 24 (IQR: 21-26), Table 3. They reported having worked a median of five harvests (IQR: 2-9). At baseline, $9 \%$ had hypertension and no one reported having diabetes.

\section{Hydration status and electrolyte solution intake}

As shown in Table 3, the percent change in body weight among the production workers was slightly negative in February and March and slightly positive in April. Results from the pre-shift urinary specific gravity show that $75 \%$ of these workers started their work shift hydrated in February and March. In April, all workers started the work shift hydrated. In notable contrast to the cane cutters, only half of production workers finished their work shift hydrated in February, $62 \%$ in March, and 77\% in April. Workers reported drinking $500 \mathrm{ml}$ to one liter of electrolyte solution and drinking a median of five liters of water.

\section{Cumulative incidence of AKI and kidney function}

Among the 99 production workers, 97 workers (98\%) had at least one episode of AKI. One-quarter of the workers (22\%) had only one AKI, $35 \%$ had two AKIs, and $40 \%$ had three AKIs. AKI cumulative incidence across the work shift was $81 \%$ in February, 63\% in March, and 77\% in April (Table 4). The percentage of workers with a stage 2 AKI went from 30 to $16 \%$ across the study time points, coinciding with an improvement in availability of water at the later time point, as discussed below.

The median eGFR at baseline was $116 \mathrm{ml} / \mathrm{min} / 1.73 \mathrm{~m}^{2}$ and the median pre-shift eGFR near 130 at all three time points. Over $90 \%$ of the workers had a normal eGFR at the start of the work shift $(\geq 90)$. Four workers had a pre-shift eGFR $<60$ and none of the workers had an eGFR $<60$ at two or more time points.

\section{Physical work intensity}

Work shift hours were approximately eight hours at each time point, including rest breaks. Workers reported taking a median of two rest breaks that were more than 15 min.

\section{Behavioral risk factors and symptoms}

Four percent or less of the workers reported smoking a cigarette the day of the study, taking a NSAID in the last $24 \mathrm{~h}$, or consuming alcoholic beverages the night before. Over half of the workers reported drinking a sugary beverage on the day of the study in February, 38\% in March, and 50\% in April. Very few workers reported symptoms on the study days. In February, 9\% of the workers reported dry mouth, 3\% reported lower back pain, and $2 \%$ reported upper back pain. These symptoms decreased in March and April.

c. Analysis of risk factors

Table 4 Kidney function of the production workers at the three study time points in Guatemala, 2017

\begin{tabular}{|c|c|c|c|}
\hline $\begin{array}{l}\text { Kidney function } \\
\text { Pre-shift }\end{array}$ & $\begin{array}{l}\text { February } \\
n=98\end{array}$ & $\begin{array}{l}\text { March } \\
n=95\end{array}$ & $\begin{array}{l}\text { April } \\
n=95\end{array}$ \\
\hline Creatinine, $\mu \mathrm{mol} / \mathrm{L}$, median (IQR) & $55.69(42.43,66.30)$ & $56.58(51.27,68.95)$ & $54.81(47.74,64.53)$ \\
\hline eGFR, $\mathrm{ml} / \mathrm{min} / 1.73 \mathrm{~m}^{2}$, median (IQR) & $133.14(118.73,147.35)$ & $129.91(113.18,140.62)$ & $130.60(115.53,143.27)$ \\
\hline \multicolumn{4}{|l|}{ Kidney disease stages, $n(\%)$} \\
\hline$>90$ eGFR & $92(94 \%)$ & $86(91 \%)$ & $92(97 \%)$ \\
\hline 60-90 eGFR & $5(5 \%)$ & $6(6 \%)$ & $3(3 \%)$ \\
\hline 30-60 eGFR & $1(1 \%)$ & $3(3 \%)$ & 0 \\
\hline 15-30 eGFR & 0 & 0 & 0 \\
\hline \multicolumn{4}{|l|}{ Cross-shift } \\
\hline Change in creatinine, median (IQR) & $0.45(0.33,0.55)$ & $0.34(0.19,0.44)$ & $0.37(0.29,0.54)$ \\
\hline Acute kidney injury ${ }^{\mathrm{a}}, n(\%)$ & $79(81 \%)$ & $60(63 \%)$ & $73(77 \%)$ \\
\hline \multicolumn{4}{|l|}{ AKI categories ${ }^{\mathrm{b}}, n(\%)$} \\
\hline Stage 1 & $54(68 \%)$ & $56(93 \%)$ & $60(82 \%)$ \\
\hline Stage 2 & $24(30 \%)$ & $4(7 \%)$ & $12(16 \%)$ \\
\hline Stage 3 & $1(1 \%)$ & 0 & $1(1 \%)$ \\
\hline
\end{tabular}

$e G F R$ estimated glomerular filtration rate, IQR interquartile range

${ }^{\mathrm{a}}$ Increase of post-shift creatinine by $\geq 26.5 \mu \mathrm{mol} / \mathrm{L}$ or 1.5 times pre-shift creatinine

${ }^{\mathrm{b}}$ Stage 1: $1.5-1.9$ times pre-shift or $\geq 26.5 \mu \mathrm{mol} / \mathrm{L}$ increase. Stage 2: 2-2.9 times pre-shift. Stage 3: 3 times pre-shift or $354 \mu \mathrm{mol} / \mathrm{L}$ increase 
Table 5 Mixed-effects logistic regression univariate analyses assessing the odds of acute kidney injury in sugarcane workers in Guatemala, $2017(N=517)$

\begin{tabular}{|c|c|c|}
\hline Characteristics & OR $(95 \% \mathrm{CI})$ & $p$ value \\
\hline \multicolumn{3}{|l|}{ Baseline } \\
\hline Age & $1.01(1,1.03)$ & 0.04 \\
\hline Local home residence (ref: Highland) & $1.13(0.89,1.44)$ & 0.32 \\
\hline Body mass index & $0.99(0.95,1.04)$ & 0.75 \\
\hline Hypertension (ref: no) & $1.37(0.68,2.77)$ & 0.37 \\
\hline Previous harvests & $1.01(0.99,1.03)$ & 0.45 \\
\hline Baseline eGFR & $0.98(0.98-0.99)$ & $<\mathbf{0 . 0 1}$ \\
\hline \multicolumn{3}{|l|}{ Hydration and electrolytes } \\
\hline Body weight, percent change & $1.01(0.98,1.04)$ & 0.55 \\
\hline Pre-shift specific gravity (per 0.01 ) & $1.41(1.19,1.67)$ & $<\mathbf{0 . 0 1}$ \\
\hline Post-shift specific gravity (per 0.01 ) & $1.48(1.27,1.72)$ & $<\mathbf{0 . 0 1}$ \\
\hline Specific gravity, percent change & $1.07(0.93,1.22)$ & 0.34 \\
\hline $\begin{array}{l}\text { Electrolyte solution intake (per } 1 \\
\text { packet) }\end{array}$ & $0.89(0.85,0.93)$ & $<\mathbf{0 . 0 1}$ \\
\hline \multicolumn{3}{|l|}{ Physical work intensity } \\
\hline Rest breaks (per 1 break) & $0.83(0.75,0.93)$ & $<\mathbf{0 . 0 1}$ \\
\hline Work shift hours (per $1 \mathrm{~h}$ ) & $0.89(0.83,0.95)$ & $<0.01$ \\
\hline Productivity, prior day (per 1 ton) ${ }^{a}$ & $1.00(0.94,1.06)$ & 0.90 \\
\hline Productivity, study day (per 1 ton) ${ }^{a}$ & $1.00(0.95,1.06)$ & 0.93 \\
\hline $\begin{array}{l}\text { Productivity } z \text {-scores (per } 1 \text { std. devia- } \\
\text { tion) }\end{array}$ & $0.93(0.82,1.05)$ & 0.25 \\
\hline Production job type (ref: cane cutter) & $3.10(2.29,4.19)$ & $<\mathbf{0 . 0 1}$ \\
\hline \multicolumn{3}{|l|}{ Heat exposure } \\
\hline Average WBGT (per $1^{\circ} \mathrm{C}$ ) & $0.90(0.82,0.98)$ & 0.02 \\
\hline Maximum WBGT (per $1{ }^{\circ} \mathrm{C}$ ) & $0.89(0.82,0.96)$ & $<\mathbf{0 . 0 1}$ \\
\hline \multicolumn{3}{|l|}{ Study day behaviors } \\
\hline Sugary beverage intake (per 1 drink) & $1.06(0.9,1.24)$ & 0.48 \\
\hline Smoked cigarette (ref: no) & $1.36(0.79,2.35)$ & 0.27 \\
\hline NSAID use (ref: no) & $1.44(0.84,2.47)$ & 0.18 \\
\hline Alcohol intake (per 1 drink) & $0.95(0.76,1.19)$ & 0.66 \\
\hline
\end{tabular}

Bold values are significant at $p<0.05$

$O R$ odds ratio, $C I$ confidence interval, $e G F R$ estimated glomerular filtration rate, WBGT wet bulb globe temperature, NSAID non-steroidal anti-inflammatory drug

${ }^{a}$ Cane cutters only, $n=418$

Table 5 summarizes the results of the univariate analysis. We found associations between AKI and several factors including age (odds ratio (OR) $1.01,95 \%$ confidence interval (CI) 1.00-1.03), baseline eGFR (OR 0.98, 95\% CI $0.98-0.99$ ), pre-shift urinary-specific gravity (OR 1.41, $95 \%$ CI 1.19-1.67), post-shift specific gravity (OR $1.48 ; 95 \%$ CI $1.27-1.72$ ), electrolyte solution intake (OR $0.89,95 \%$ CI $0.85-0.93$ ), rest breaks (OR $0.83,95 \%$ CI $0.75-0.93$ ), work shift hours (OR 0.89, 95\% CI 0.83-0.95), job type (OR 3.10, 95\% CI 2.29-4.19), and average and maximum WBGT (OR $0.90,95 \%$ CI $0.82-0.98$ and OR 0.89, 95\% CI 0.82-0.96). Higher WBGT and more work shift hours were associated
Table 6 Mixed-effect multivariable regression analyses showing odds ratios $(95 \% \mathrm{CI})$ for risk factors for acute kidney injury in sugarcane workers in Guatemala, $2017(N=517)$

\begin{tabular}{llr}
\hline Covariates & OR (95\% CI) & $p$ value \\
\hline Age & $0.99(0.97-1.01)$ & 0.22 \\
Hypertension (ref: no) & $1.10(0.56-2.16)$ & 0.79 \\
Baseline eGFR & $0.98(0.97-0.99)$ & $<\mathbf{0 . 0 1}$ \\
Work shift hours & $0.98(0.90-1.06)$ & 0.60 \\
Rest breaks & $0.99(0.86-1.14)$ & 0.94 \\
Electrolyte solution (per packet) & $0.94(0.89-0.99)$ & $\mathbf{0 . 0 4}$ \\
Pre-shift specific gravity (per 0.01) & $1.21(0.99-1.45)$ & 0.05 \\
Post-shift specific gravity (per 0.01) & $1.24(1.02-1.52)$ & $\mathbf{0 . 0 3}$ \\
Average WBGT (per $\left.1{ }^{\circ} \mathrm{C}\right)$ & $0.93(0.81-1.06)$ & 0.25 \\
\hline
\end{tabular}

Bold values are significant at $p<0.05$

$C I$ confidence interval, $e G F R$ estimated glomerular filtration rate, $W B G T$ wet bulb globe temperature

with a lower likelihood of developing an AKI. In a post hoc analysis, we examined this relationship and other possible factors that may be correlated with WBGT, including work shift hours, post-shift specific gravity, electrolyte intake, and productivity. Work shift hours were inversely associated with WBGT for cane cutters (as WBGT increased, work shift hours decreased: $r=-0.31, p<0.01$ ). Electrolyte solution intake was positively correlated with WBGT for both groups (as WBGT increased, electrolyte intake increased: cane cutters $r=0.28, p<0.01$ and production workers, $r=0.73, p<0.01)$. Post-shift specific gravity and productivity were not correlated with WBGT.

Table 6 shows the overall associations between examined risk factors and AKI in the multivariable regression model. We observed that higher post-shift specific gravity was associated with greater odds of developing an AKI (OR $1.24,95 \%$ CI 1.02-1.52). Higher baseline eGFR and higher electrolyte solution intake were associated with lower odds of developing an AKI (OR 0.98, 95\% CI 0.97-0.99 and OR 0.94, 95\% CI 0.89-0.99, respectively). Higher pre-shift specific gravity approached statistical significance (OR 1.21, 95\% CI 0.99-1.46). Next, we explored interaction terms independently to assess the compounding effect of dehydration and certain behaviors that have been hypothesized as contributors to kidney function decline; these included sugary beverage intake, smoking, and NSAID use. Only the interaction term between post-shift specific gravity and NSAID use was significant. Workers who used NSAIDs and had increasing specific gravity were 8.38 times more likely to experience an AKI (OR 8.38, 95\% CI 1.67-42.16). The confidence interval is wide for this interaction term due to the low number of workers that reported using NSAID and the low number of workers with elevated specific gravity. The separate interaction terms with post-shift specific gravity and smoking and sugary beverage intake were not 
significant (OR 1.26, 95\% CI 0.48-3.29 and OR 0.92, 95\% CI $0.75-1.14$, respectively). When we included the NSAID $\mathrm{x}$ post-shift specific gravity in the multivariable model, this interaction term remained significant, as did baseline eGFR and electrolyte solution intake (OR 6.05, 95\% CI 1.49-24.62, OR 0.98, 95\% CI 0.97-0.99, and OR 0.94, $95 \%$ CI $0.88-0.99$, respectively). Pre-shift specific gravity became significant in this model and post-shift specific gravity approached significance (OR 1.21, 95\% CI 1.00-1.46 and OR $1.20,95 \%$ CI $0.98-1.47$ ). While the interaction term is significant, there is high uncertainty in the estimate due to the wide confidence interval.

\section{Discussion}

In this study, we evaluated 517 Guatemalan sugarcane workers to understand the effects of hydration, high physical workload, heat exposure and behavioral risk factors on AKI. A key discovery is that we observed a surprisingly high incidence of cross-shift AKI even when workers were well hydrated. Despite appropriate hydration in the field, increasing post-shift specific gravity was observed to be a risk factor for AKI and higher electrolyte solution intake was found to be protective. The fact that $81 \%$ of these sugarcane workers demonstrated evidence of at least one AKI during the study period demonstrates a higher vulnerability than has previously been reported in agricultural workers from the U.S. (Mix et al. 2017; Moyce et al. 2017).

These findings have practical public health implications. First, enhanced hydration, including use of electrolyte solution, is important to mitigate the risk of AKI in agricultural workers. Additionally, other factors appear to contribute to AKI risk in those who are dehydrated. Increasing postshift specific gravity in combination with NSAID use was found to be a significant risk factor for AKI across the work shift. Therefore, the effect of NSAID use on AKI appears to be dependent on hydration status; however, these findings should be interpreted with caution due to the small overall presence of these events. These data support, but do not prove, the hypothesis that dehydrated individuals are at greater risk of harm from nephrotoxins (Correa-Rotter et al. 2014; Weiner et al. 2013).

While the present study did not find a direct association between increasing WBGT and AKI, heat stress cannot be ignored, since WBGT was over $30^{\circ} \mathrm{C}$ on almost all study days with little variation. As expected, we observed that as WBGT increased, cane cutters shift hours decreased. This may explain why increasing WBGT was not associated with AKI. We used a single point measure, field-based WBGT, for the all the workers on each study day. Future studies should measure individual-level heat exposure. Several other studies have also observed cross-shift increase in serum creatinine under conditions of high heat stress and dehydration (Garcia-Trabanino et al. 2015; Mix et al. 2017; Moyce et al. 2017). A study in El Salvador, among 189 sugarcane cutters, found a significant increase in serum creatinine across the work shift which was positively associated with temperature and heat strain (Garcia-Trabanino et al. 2015). In the U.S., the relationship between AKI and heat stress has also been investigated. One study conducted in California with 283 agricultural workers observed a 12\% incidence of AKI over a work shift and found a link between kidney injury and temperature (Moyce et al. 2017). Workers who experienced heat strain, evaluated on core body temperature and heart rate, had $34 \%$ increased risk of AKI. In a study conducted in Florida, 33\% of the 192 agricultural workers had at least one AKI. For each 5 degree ${ }^{\circ} \mathrm{F}$ increase in heat index, odds of an AKI increased 37\% (Mix et al. 2017). These workers had much higher pre- and post-shift urinary specific gravity than the workers in our study, with $53 \%$ of their participants demonstrating a pre-shift specific gravity $\geq 1.020$ and $81 \%$ demonstrating a post-shift specific gravity $\geq 1.020$.

In addition, we found that workers who reported higher electrolyte solution intake were less likely to develop an AKI. A study conducted in Nicaragua found that for every additional electrolyte solution packet consumed by cane cutters, mean late-harvest eGFR was $6.1 \mathrm{ml} / \mathrm{min} / 1.73 \mathrm{~m}^{2}$ higher (Laws et al. 2015). One electrolyte of particular interest is potassium. For example, decrease in serum potassium levels across the work shift have been observed in agricultural workers with heat stress and dehydration (Garcia-Trabanino et al. 2015). In addition to potassium, changes in serum sodium may play a role in the development of AKI. A study conducted in Brazil among sugarcane workers found a negative correlation between the changes in serum creatinine and the changes in serum sodium across the work shift (Santos et al. 2015). Authors suggest that decrease in sodium could worsen volume depletion due to a decrease in plasma osmolality, which may explain why electrolyte solution appears to confer a protective effect against kidney injury.

Our study has several strengths. It is the first study that examines cross-shift creatinine on three workdays distributed throughout the harvest season in such a large cohort of workers. Second, we measured clinically important biomarkers for hydration status and kidney function and collected detailed information on risk factors at three separate time points. In addition, we were able to optimize hydration status among workers through an enhanced WRS intervention. Nevertheless, several limitations need to be acknowledged. Based on our previous study comparing post-shift POC creatinine to venipuncture measures, we applied a correction factor to pre- and post-shift POC creatinine measures (Griffin et al. 2018). Further work is required to determine if the correction factor is accurate for pre-shift measures. There 
are several other potential mechanisms for the development of AKI that were not studied, such as rhabdomyolysis and inflammation. In addition, there are other potentially nephrotoxic substances that we did not study for their contributions to AKI risk (Johnson et al. in press). Although it is theoretically possible that exertional rhabdomyolysis may have contributed to an increase in post-shift serum creatinine and affected our estimate of AKI, we think this is unlikely for three reasons. First, if rhabdomyolysis was a main contributor to AKI, it would have been expected that individuals that were more productive (i.e., cut more cane) would have incurred higher levels of muscle damage and therefore would demonstrate higher post-shift serum creatinine. This was not the case. Second, in a separate study, we measured post-shift serum creatine kinase (CK) in a subset of this current study population, 105 sugarcane cutters, during the same three time points, and observed only 2.5 - to threefold increase of CK above baseline (Sorensen et al. 2019). In that group, there was no statistically significant association between CK level and post-shift serum creatinine. Third, in a study of 203 healthy individuals with exercise-induced elevation of CK, Clarkson et al. observed no rise in serum creatinine, despite CK levels 3.1- to 6.2-fold above baseline, at 4,7 , and 10 days post-exercise (Clarkson et al. 2006).

We acknowledge that there are more sensitive and precise indicators of physical exertion, such as heart rate, that can be used instead of tons cut and work shift hours. In an effort to improve precision, we calculated each worker's productivity $z$-score to compare their study physical exertion to their average level of exertion.

An additional limitation is that it can be difficult to determine if workers are truly hydrated using specific gravity, since as kidney function declines, urine concentrating capacity is reduced (Zittema et al. 2017). We were unable to measure exactly when the fluid was consumed. While the specific gravity is surprisingly low in this group of workers, the low urine-specific gravity reflects the high water intake and is supported by the presence of other measures of hydration that were collected, and by absence of weight loss. Cane cutters reported drinking a median of 15 liters of water per day; these workers had very low specific gravity. Production workers reported drinking a median of $5 \mathrm{~L}$ of water per day and appropriately had higher specific gravity. Specific gravity was also measured using urine dipsticks for all participants and yielded very similar results compared to refractometer measures. Among the workers with a refractometer specific gravity of $<1.005,98 \%$ had a dipstick specific gravity of less than or equal to 1.010 . In our separate study of 105 sugarcane cutters (Sorensen et al. 2019), by calculating serum osmolality, we found that hypo-osmolality was common, with more than $30 \%$ of workers demonstrating a serum osmolality $<280 \mathrm{mmol} / \mathrm{L}$ and an additional $50 \%$ were well hydrated ( 280 to $295 \mathrm{mmol} / \mathrm{L}$ ).
Fewer than $2 \%$ of the workers demonstrated hyperosmolality (osmolality $>295 \mathrm{mmol} / \mathrm{L}$ ).

Another limitation is related to the self-reported data collection of some of the risk factors including NSAID use and electrolyte intake. In an effort to minimize misclassification bias of the NSAIDs, workers were shown pictures of the over-the-counter products. NSAID use may be underreported, since Pantaleon has discouraged, but not restricted, their use. In addition, biomarkers were only collected on 3 days per worker, and we cannot exclude the possibility that workers drank more water and electrolyte solution on the study days. We were not able to follow workers after the end of the harvest to determine whether or not they eventually developed CKDu.

In conclusion, our results demonstrate that a large proportion of workers are experiencing repeat AKI, under intense heat conditions. While hydration and electrolyte intake are found to be protective, they do not prevent all injuries. This suggests that other factors may be contributing to injury that could develop into chronic damage and CKDu over time.

Funding This evaluation was supported in part by Pantaleon; the Chancellor, CU Anschutz; NIH NIEHS (R21 ES028826); Centers for Disease Control and Prevention/National Institute for Occupational Safety and Health (U19 OH01127); and Mountain and Plains Education and Research Center (T42 OH009229).

\section{Compliance with ethical standards}

Conflict of interest Pantaleon and the University of Colorado have a memorandum of understanding and a contract for consulting on Total Worker Health programs. It supports part of the salaries for the Center for Health, Work, \& Environment authors (JBD, LK, MD, LT, and LN). University of Colorado and Pantaleon are separate, independent organizations. University of Colorado employed appropriate research methods in keeping with principles of academic freedom, based conclusions on critical analysis of the evidence, and reported findings fully and objectively. The terms of this arrangement have been reviewed and approved by the University of Colorado Denver in accordance with its conflict of interest policies. Authors: HY, CS, RJ, CA, AC, EJ, EC, and EA have no conflict of interest to report.

Ethics approval Ethics review and approval for the study was done by the Colorado Multiple Institution Review Board (COMIRB) and in Guatemala by the Comite de Etica, Facultad de Medicina, Universidad Francisco Marroquin-Hospital Universitario Esperanza. All participants provided written informed consent at time of enrollment.

Open Access This article is distributed under the terms of the Creative Commons Attribution 4.0 International License (http://creativeco mmons.org/licenses/by/4.0/), which permits unrestricted use, distribution, and reproduction in any medium, provided you give appropriate credit to the original author(s) and the source, provide a link to the Creative Commons license, and indicate if changes were made. 


\section{References}

Bodin T, Garcia-Trabanino R, Weiss I, Jarquin E, Glaser J, Jakobsson $\mathrm{K}$ et al (2016) Intervention to reduce heat stress and improve efficiency among sugarcane workers in El Salvador: Phase 1. Occup Environ Med 73(6):409-416

Butler-Dawson J, Krisher L, Asensio C, Cruz A, Tenney L, Weitzenkamp D et al (2018) Risk factors for declines in kidney function in sugarcane workers in Guatemala. J Occup Environ Med 60(6):548-558

Clarkson PM, Kearns AK, Rouzier P, Rubin R, Thompson PD (2006) Serum creatine kinase levels and renal function measures in exertional muscle damage. Med Sci Sports Exerc 38(4):623-627

Correa-Rotter R, Wesseling C, Johnson RJ (2014) CKD of unknown origin in central america: the case for a mesoamerican nephropathy. Am J Kidney Dis 63(3):506-520

Crowe J, Wesseling C, Solano BR, Umana MP, Ramirez AR, Kjellstrom $\mathrm{T}$ et al (2013) Heat exposure in sugarcane harvesters in Costa Rica. Am J Ind Med 56(10):1157-1164

Garcia-Arroyo FE, Cristobal M, Arellano-Buendia AS, Osorio H, Tapia E, Soto V et al (2016) Rehydration with soft drink-like beverages exacerbates dehydration and worsens dehydration-associated renal injury. Am J Physiol Regul Integr Comp Physiol 311(1):R57-R65

Garcia-Trabanino R, Jarquin E, Wesseling C, Johnson RJ, GonzalezQuiroz M, Weiss I et al (2015) Heat stress, dehydration, and kidney function in sugarcane cutters in EI Salvador-a cross-shift study of workers at risk of Mesoamerican nephropathy. Environ Res 142:746-755

Glaser J, Lemery J, Rajagopalan B, Diaz HF, Garcia-Trabanino R, Taduri $\mathrm{G}$ et al (2016) Climate change and the emergent epidemic of CKD from heat stress in rural communities: the case for heat stress nephropathy. Clin J Am Soc Nephrol 11(8):1472-1483

Griffin BR, Butler-Dawson J, Dally M, Krisher L, Cruz A, Weitzenkamp D et al (2018) Unadjusted point of care creatinine results overestimate acute kidney injury incidence during field testing in Guatemala. Plos One 13:9

Hahn K, Kanbay M, Lanaspa MA, Johnson RJ, Ejaz AA (2017) Serum uric acid and acute kidney injury: a mini review. J Adv Res 8(5):529-536

Hodgson L, Walter E, Venn R et al (2017) Acute kidney injury associated with endurance events-is it a cause for concern? A systematic review. BMJ Open Sport \& Exercise Medicine 3:e000093. https://doi.org/10.1136/bmjsem-2015-000093

Improving Global Outcomes Acute Kidney Injury Work Group (2012) Kidney disease. In: Clinical practice guideline for acute kidney injury, vol 2. Kidney Inter, Suppl, pp 1-138

Johnson RJ, Wesseling C, Newman LS (in press) Chronic kidney disease of unknown cause in agricultural communities. N Engl J Med

Laws RL, Brooks DR, Amador JJ, Weiner DE, Kaufman JS, Ramirez-Rubio $\mathrm{O}$ et al (2015) Changes in kidney function among Nicaraguan sugarcane workers. Int J Occup Environ Health 21(3):241-250

Laws RL, Brooks DR, Amador JJ, Weiner DE, Kaufman JS, RamirezRubio O et al (2016) Biomarkers of kidney injury among nicaraguan sugarcane workers. Am J Kidney Dis 67(2):209-217

Levey AS, Stevens LA (2010) Estimating GFR using the CKD epidemiology collaboration (CKD-EPI) creatinine equation: more accurate GFR estimates, lower CKD prevalence estimates, and better risk predictions. Am J Kidney Dis 55(4):622-627

Lunyera J, Mohottige D, Von Isenburg M, Jeuland M, Patel UD, Stanifer JW (2016) CKD of uncertain etiology: a systematic review. Clin J Am Soc Nephrol 11(3):379-385

Mix J, Elon L, Mac VVT, Flocks J, Economos E, Tovar-Aguilar AJ et al (2018) Hydration status, kidney function, and kidney injury in Florida agricultural workers. J Occup Environ Med 60(5):E253-E260

Moyce S, Mitchell D, Armitage T, Tancredi D, Joseph J, Schenker M (2017) Heat strain, volume depletion and kidney function in California agricultural workers. Occup Environ Med 74(6):402-409

OSHA (2017) OSHA technical manual. Occupational Safety and Health Administration. Section III: Chap. 4

Perrier ET, Bottin JH, Vecchio M, Lemetais G (2017) Criterion values for urine-specific gravity and urine color representing adequate water intake in healthy adults. Eur J Clin Nutr 71(4):561-563

Roncal-Jimenez C, Lanaspa MA, Jensen T, Sanchez-Lozada LG, Johnson RJ (2015) Mechanisms by which dehydration may lead to chronic kidney disease. Ann Nutr Metab 66:10-13

Santos UP, Zanetta DMT, Terra-Filho M, Burdmann EA (2015) Burnt sugarcane harvesting is associated with acute renal dysfunction. Kidney Int 87(4):792-799

Sorensen CJ, Butler-Dawson J, Dally M, Krisher L, Griffin BR, Johnson RJ et al (2019) Risk factors and mechanisms underlying crossshift decline in kidney function in Guatemalan sugarcane workers. J Occup Environ Med 61(3):239-250

Speeckaert MM, Delanghe JR, Vanholder RC (2013) Chronic nicotine exposure and acute kidney injury: new concepts and experimental evidence. Nephrol Dial Transpl 28(6):1329-1331

Vos T, Abajobir AA, Abbafati C, Abbas KM, Abate KH, Abd-Allah F et al (2017) Global, regional, and national incidence, prevalence, and years lived with disability for 328 diseases and injuries for 195 countries, 1990-2016: a systematic analysis for the Global Burden of Disease Study 2016. Lancet 390(10100):1211-1259

Watts N, Adger WN, Ayeb-Karlsson S, Bai YQ, Byass P, CampbellLendrum D et al (2017) The Lancet Countdown: tracking progress on health and climate change. Lancet 389(10074):1151-1164

Webb MC, Salandy ST, Beckford SE (2016) Monitoring hydration status pre- and post-training among university athletes using urine color and weight loss indicators. J Am Coll Health 64(6):448-455

Wegman DH, Apelqvist J, Bottai M, Ekstrom U, Garcia-Trabanino R, Glaser J et al (2018) Intervention to diminish dehydration and kidney damage among sugarcane workers. Scand J Work Environ Health 44(1):16-24

Weiner DE, McClean MD, Kaufman JS, Brooks DR (2013) The Central American epidemic of CKD. Clin J Am Soc Nephrol 8(3):504-511

Zittema D, Casteleijn NF, Bakker SJL, Boesten LSM, Duit AAM, Franssen CFM et al (2017) Urine concentrating capacity, vasopressin and copeptin in ADPKD and IgA nephropathy patients with renal impairment. Plos One 12(1):14

Publisher's Note Springer Nature remains neutral with regard to jurisdictional claims in published maps and institutional affiliations. 


\section{Affiliations}

\section{Jaime Butler-Dawson ${ }^{1,2,3}$ - Lyndsay Krisher ${ }^{1,2} \cdot$ Hillary Yoder $^{4} \cdot$ Miranda Dally $^{1,2,3} \cdot$ Cecilia Sorensen $^{2,5}$. \\ Richard J. Johnson ${ }^{2,6}$. Claudia Asensio ${ }^{7}$ - Alex Cruz ${ }^{7}$ Evan C. Johnson ${ }^{4}$. Elizabeth J. Carlton ${ }^{2,3}$ • Liliana Tenney ${ }^{1,2,3}$. Edwin J. Asturias ${ }^{8,9,10} \cdot$ Lee S. Newman $12,3,11$}

1 Department of Environmental and Occupational Health, Center for Health, Work, and Environment Colorado School of Public Health, University of Colorado, Anschutz Medical Campus 13001 E. 17th Pl., Ste. W3111, Aurora, CO 80045, USA

2 Colorado Consortium on Climate Change and Human Health, University of Colorado, Anschutz Medical Campus, Aurora, CO, USA

3 Department of Environmental and Occupational Health, School of Public Health, University of Colorado, Anschutz Medical Campus, Aurora, CO, USA

4 Department Kinesiology and Health, University of Wyoming, Laramie, WY, USA

5 Department of Emergency Medicine, University of Colorado School of Medicine, Anschutz Medical Campus, Aurora, CO, USA
6 Division of Renal Diseases and Hypertension, School of Medicine, University of Colorado, Anschutz Medical Campus, Aurora, CO, USA

7 Pantaleon, Guatemala City, GT, USA

8 Division of Pediatric Infectious Diseases, School of Medicine, University of Colorado, Anschutz Medical Campus, Aurora, CO, USA

9 Center for Global Health, Colorado School of Public Health, Anschutz Medical Campus, Aurora, CO, USA

10 Department of Epidemiology, Colorado School of Public Health, University of Colorado, Anschutz Medical Campus, Aurora, CO, USA

11 Division of Pulmonary Sciences and Critical Care Medicine, Department of Medicine, University of Colorado School of Medicine, Anschutz Medical Campus, Aurora, CO, USA 